\title{
Introduction to Modeling of Buying Decisions
}

\author{
O. Grünwald
}

\begin{abstract}
Buying decision models of customers to adjust the competitiveness of organizations have been a challenge for marketing disciplines for several generations. This topic has been explored by researchers and academics in past years, and quite an extensive theoretical base exists with a number of approaches for dealing with this challenge.

This paper presents some approaches for creating a customer decision model, and provides experimental results from an electronic investigation intended to build the Kano Model; to prove an ability to understand the modeling principle; and to find out the interpretation of the examined demand in a specific market segment involving students of a technical university. The last section of the paper contains a brief introduction to Choice-Based Modeling with Choice-Based Conjoint Analysis (CBC), which was tailored for modeling purchasing decisions.
\end{abstract}

Keywords: customer behaviors, Kano model, choice-based conjoint analysis.

\section{Models of Customer Behaviors}

Modeling purchasing decisions is derived from general behavioral theory, according to which customer decisions are influenced primarily by cultural factors, social factors and personal factors [1]. Buying decision models seek the best mathematical way to emulate the reality of markets. Statistical surveys to obtain input data are usually performed with a sample of the population. Models for understanding customer purchasing behavior are usually based on the stimulus-response principle. Influences in the buying decision process are shown in Figure 1, where the final decision is shaped by incentives of competitive bids and personal characteristics of the buyers.

During a marketing interview, respondents receive incentives with the specific information that the

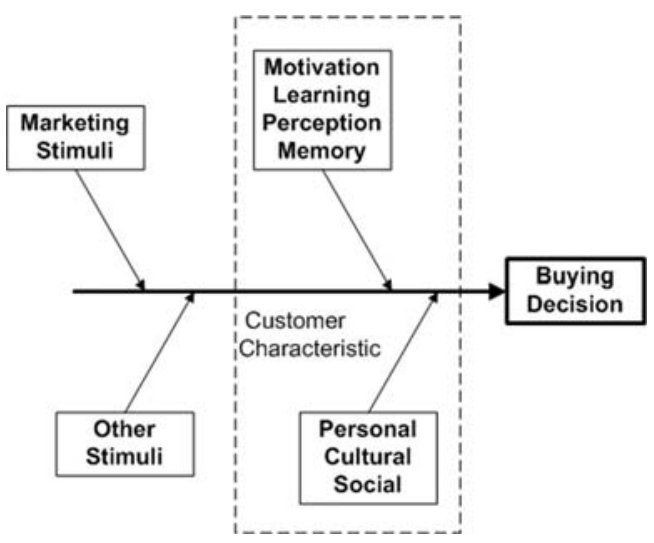

Fig. 1: Influences of external and personal factors in the buying decision process respondents understand. Then the incentive is transformed to a perception which further enters the human consciousness. A psychological process together with the personal characteristics of the respondents arising from the perception forms the resulting purchasing decision. Kotler describes four key processes constituting the final decision: the motivation process, the perception process, the learning process and the memory process.

Marketers consider motivating factors responsible for the purchase decision to be particularly important. The motivational process affecting customers' decisions is described in the literature by three theories: Freudian theory, Maslow theory and Herzberg theory.

Freudian theory assumes that the forces constituting human behavior are for the most part unconscious. People do not fully understand their own motivations for making decisions, such as the choice of a specific bundle of product features. To identify and track the motivation of people, the laddering technique [2] is used, which is in accordance with the means end chain theory. It is employed to model elements of three types: attributes, consequences and basic values. The technique monitors and analyzes connections and consequences between these elements, which can be represented by a summary table with counts of connections. The dominant connections can be represented in a tree diagram. The result then represents the dominant perceptual orientations (ways of thinking) across consumers with respect to the product category, and can be represented by a hierarchical value map (HVM). This is essentially a tree diagram with a hierarchical structure of linkages or associations across levels of abstraction. 
Maslow theory classifies the needs of customers into five hierarchical levels. According to this theory, people are driven by needs that they want to meet at a given moment. The hierarchy of needs in the following list reflects a priority sequence from highest to lowest level according to the traditional pyramid scheme:

1. Self-actualization Needs

2. Esteem Needs

3. Social Needs

4. Safety Needs

5. Psychological Needs.

The needs belonging to the fifth and lowest psychological level, e.g. the need to drink, are the basal needs to ensure vital functions of human life, and must be satisfied before fulfilment of the needs from the higher levels. The highest category of the selfactualization level of needs contains needs as selfdeployment and realization [1].

Herzberg theory deals with two types of factors. The first type is the group of dissatisfiers. These factors cause dissatisfaction among customers, and they want to avoid them. Although these properties do not sell a product, they certainly cause a customer not to buy the product. The other type of factors is the group of satisfiers, which in turn are sought by the customers and ensure customer satisfaction in proportion to their performance. In a competitive environment, these properties allow diversification of the product against other products designed to meet similar needs in a specific customer segment.

\section{Kano Model}

The Kano model (Kano 1984) is designed for marketing surveys. It extends Herzberg's theory from two factors to three factors classifying the needs of customers. The first category of needs in the model comprises mandatory must-be requirements. If the product or service does not meet these requirements, customers will be very dissatisfied because the product cannot fulfill its purpose well without them. On the other hand, when these requirements meet the customers' needs, they are perceived as self-evident and they do not cause any customer satisfaction, as in Herzberg's theory. The second type is a class of one-dimensional needs. Their increasing compliance in a product will proportionally increase the customer satisfaction gained from the experience of using it. These requirements are usually of a parametrical type (for example, a performance, a consumption or a durability). The third type is a class containing attractive needs. These requirements have the greatest influence on how satisfied customers will be with a product. Customers are mostly unable to specify attractive needs by themselves, because these requirements are often unconscious. A higher level of their compliance will also increase customer satisfaction more than proportionately. However, if attractive needs are not met, customers will not feel any dissatisfaction. Needs of this type are significant for a differentiated and positioned product.

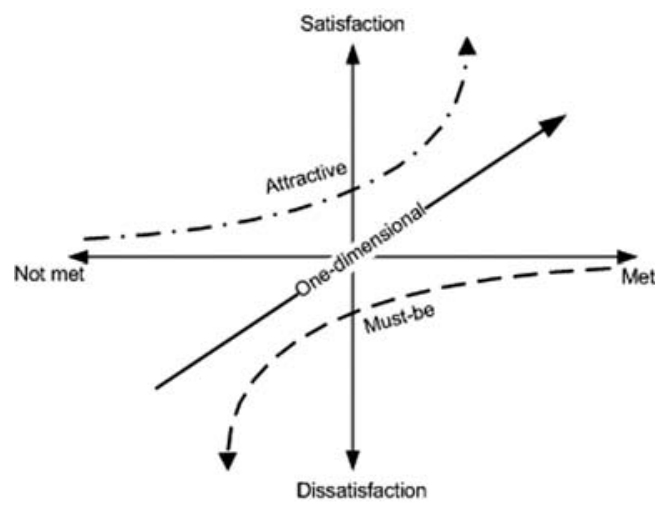

Fig. 2: Kano model, with three categories of customer needs (Must-be, One dimensional and Attractive)

The Kano model makes it possible to divide customer needs into a mandatory category, a onedimensional category and an attractive category of requirements. A product manager may determine what requirements will ensure the highest customer satisfaction, and will better understand the product offerings. He can find out about actual fulfillment of the requirements (the horizontal dimension in Figure 2) of an already established product, and can determine the importance of each individual product feature, decide which feature to prioritize and which to ignore if is not possible to meet all of them due to budget constraints. He can define the requirements that have highest potential to increase the level of product performance, or discover completely new requirements demanded by customers.

\subsection{Design of the Kano Model}

The first step in the design is to recognise which requirements it is appropriate to employ in the model. In direct questioning on motives and shopping requirements, customers usually express their conscious desires. This direct method cannot reveal the unconscious motives involved in purchasing decisions which really lead to customer satisfaction when using the product. In order to determine the requirements indirectly, it is appropriate to make interviews with the following questions [5].

1. What are the associations the customer makes when using product $\mathrm{xy}$ ?

2. What problems/defect/complaints does the customer associate when using product $\mathrm{xy}$ ?

3. What are the criteria the customer takes into account when buying product $\mathrm{xy}$ ? 
4. What features or services do customers expect? What would customers change in product $\mathrm{xy}$ ?

The first type question is often answered unclearly, but we can detect the attitudes towards the product. Answers to the second type of question define new requirements for the product that were previously unknown to the manufacturer. The third type of response will involve mainly one-dimensional (performance-related) requirements. The fourth type of question will evoke requirements that are already known, and consumers are aware about their expectations, but they have yet not been obtained in the product. To prove the Kano model, we designed an interview with 80 respondents involved in survey. The questions concerned the respondents' satisfaction with their studies at a technical university. The main requirements identified for the survey are listed in the appendix.

\subsection{Concept of a Kano Questionnaire}

A Kano questionnaire usually consists of three types of questions adopted for the purpose which we are endeavoring to find out. In order to classify the requirements into categories, the questionnaire will include paired-type questions targeted at a function/dysfunction of each requirement. Further questions that ask about the dimension of current compliance are added (in our interview, a number of these questions are variable for each requirement in order to target different subparts). The results are plotted in Figure 2 in the dimension of the horizontal axis. The third question type is intended to obtain the importance level of the requirements, which will enable us to prioritize the features for their further integration into developing services related to the studies.

A paired-type question is composed for each product property included for consideration in the survey as two questions asking for two hypothetical situations that consider the case firstly that a requirement is satisfied by the product and secondly for the situation when the requirement is not met. For each question in the pair, respondents can select one of five standardized responses. All paired questions have the same standardized options.

positive How do you assess whether you have the latest information and whether you can use a consolidated information system?

○ It is excellent

- It must be that way

- I do not care

- I can live with it

- It is a big problem

negative How do you assess whether you do not have the latest information and you cannot use a consolidated information system? $\circ$ It is excellent

- It must be that way

- I do not care

- I can live with it

- It is a big problem

The retrieved paired responses for each requirement are then classified into 6 different classes [4] according to the selected combinations.

Table 1: The classification matrix for combinations of answers collected from the paired questions

\begin{tabular}{|c|c|c|c|c|c|}
\hline Requirements & \multicolumn{5}{|c|}{ Dysfunctional $\rightarrow$} \\
\hline$\downarrow$ Functional & like & must be & neutral & live with & dislike \\
\hline like & Q & A & A & A & O \\
\hline must be & R & I & I & I & M \\
\hline neutral & R & I & I & I & M \\
\hline live with & R & I & I & I & M \\
\hline dislike & R & R & R & R & Q \\
\hline
\end{tabular}

In Table 1, the rows and the columns determine the class of requirement based on the combination of paired responses. Category A is for attractive requirements, category $\mathrm{O}$ for one-dimensional requirements, and category $\mathrm{M}$ for must be requirements. Category I means that the response combination is indifferent, and the respondent has not provided decisive information about the requirement in a combination of responses. Category R signals that an increasing level of the related requirement decreases the customer satisfaction. Category $\mathrm{Q}$ is for unclear responses with a combination without an understandable meaning, for pairs of positive and negative responses in contradiction to each other. Requirements with dominant $\mathrm{Q}$ category should be interviewed again. A combination of pair answers belonging to the Q category and to the $\mathrm{R}$ category should not occur very often, otherwise this result means improper product features or invalid responses from the respondents.

Performance questions presented in the interview together with the paired question type are about how a product currently meets the claims of respondents, and they assume that the respondents have previous experience of using the product. The answers are expressed on rating scales. In the interview we used scales with six degrees (1-7) plus one option for the case that the respondent is not affected by the request. We implemented different numbers of these questions under each individual requirement, as it was necessary to ask about specific sub-areas and related details. The questions that we used are listed in the appendix as a second level of the list. An example of a question to find out how the specific requirement currently meets the customers' (students') requirements is shown below. 
How satisfied are you with opportunities to obtain current information related to your studies?

0000000

does not affect

very

very

me

dissatisfied

satisfied

Importance questions were the third type of question in the interview. The respondents evaluated each single requirement again on a scale of 1 to 7 , and were asked to reflect the degree of importance of each single demand in accordance with their perception.

\subsection{Survey of student satisfaction at CTU}

The survey to conclude the Kano model was conducted electronically with a group of 60 students who had previously been acquainted with the principle of the model. The respondents answered the questionnaire without any assistance on their own computer. They received the instruction and an invitation to participate in the interview by an email message. Each requirement in the interview obtained 30 responses. The total set of 20 requirements was divided into two variants of the questionnaire, each with 10 steps +1 step for importance questions. This partition reduced the interviewing time and prevented an overload of respondents during the interview at the expense a smaller number of responses. The variants of the questionnaire were alternated after each new respondent. In this way, each question in the interview was responded to by approximately one half of the total number of respondents. We investigated the classification of requirements, the degree of their current performance, and their importance for the respondents.

An interpretation of the results includes for each requirement a specification of the frequency of the specific class in descending order. Some requirements were not given an entirely clear category, so the category with the highest frequency was considered as dominant. The categories in other places in the order should also be taken into account when interpreting the results, taking into consideration the distributions of the frequencies of other classes. Some of the answers were dominant in the category of attractive, and simultaneously the requirement received almost same the number of must be answers and there was no great difference between them. In this case, the absence of a such requirement in the service will reduce the satisfaction and at the same time dissatisfaction will emerge. We also calculated the coefficient of satisfaction [6] CS coefficient as shown in the following formulas.

$$
s=\frac{A+O}{A+O+I+M}
$$

Where $s$ denotes the coefficient of satisfaction. A, $O, I, M$ are the frequencies of classifications into the categories

$$
d=\frac{O+M}{(-1) \times(A+O+I+M)}
$$

Where $d$ means coefficient of dissatisfaction.

$$
C S=s+d=\frac{A-M}{A+O+I+M}
$$

$C S$ means the total coefficient of satisfactions, and emphasizes the extremes of the satisfaction. If we use an averaging procedure for the responses of each requirement, it would compensate the result to the mean values. Therefore, the CS coefficient subtracts the one dimensional responses in the numerator and then the result reflects by $+/-$ sign whether it is an attractive or must be requirement, and the size of the coefficient expresses the absolute value of the $A$ and $M$ responses to the total number of responses. The CS coefficient reflects in its inner parts $a$ and $d$ how strongly the product requirement affects the satisfaction, or the dissatisfaction, if the requirement is/is not met.

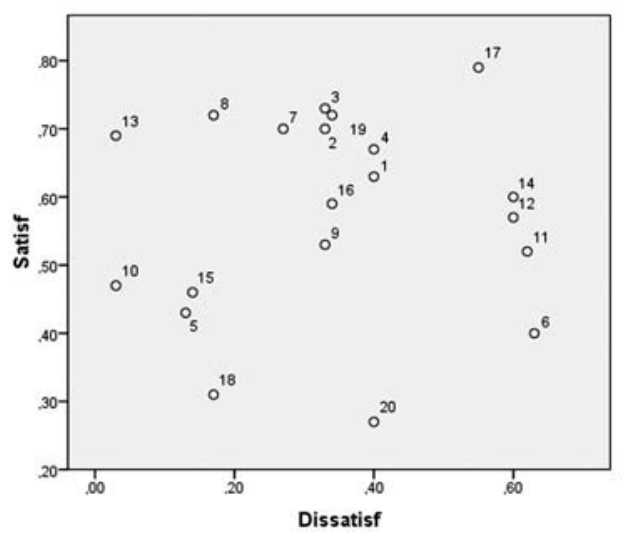

Fig. 3: Positioning of students' requirements in a two dimensional system (satisfaction and dissatisfaction effects)

Figure 3 shows the rate of satisfaction in two dimensions: $s$ as the satisfaction coefficient and $d$ as the dissatisfaction coefficient. The requirements placed on top of the coordinate system indicate the degree of satisfaction if the requirement is met, and the location of the right means the degree of dissatisfaction when not fulfilled. This figure shows that if requirement No. 13 - Use of the latest technology for teaching and opportunities to use your own notebook is not met, respondents will not feel strong dissatisfaction, but they will feel great satisfaction if they have access to good wireless connections and software support. In contrast, requirement No. $6-$ Content accordance of the field of study with world trends will cause high dissatisfaction if is not met, and no significant satisfaction if is fulfilled. 
In our questionaire, each requirement-step consisted of a paired question and a variable number of differently aimed second type questions on the current level of performance. The level of compliance was expressed by the respondents on a rating scale. When averaging the responses, all requirements were slightly above the middle of the range. From this result it was very difficult to determine the actual performance despite the compensation feature. CP Coefficient of performance was therefore calculated as an additional indicator, in order to provide an overview.

$$
C P=\frac{\sum_{i=3}^{7} f_{i}-\sum_{i=1}^{5} f_{i}}{\sum_{i=1}^{7} f_{i}}
$$

where $C P$ is the coefficient of performance, focused on the result of extreme values to avoid the compensated result, $f_{n}$ denotes the choice frequencies of each level on a seven-grade rating scale by the respondents. The result from the questionaire is shown in Figure 4. The labels A,B,C refer to the second type question. $A$ is the first performance question belonging under a specific requirement, $B$ is the second question, and $C$ is the third question. Three questions in the set was the maximum number in our questionnaire. The requirements are marked with numbers 1 to 20. The classification into categories $(M, A, O, I)$ is derived from the superior requirement and is thus identical for all variants of all related performance questions (which have the same number). The requirements are numbered from 1 to 20 .

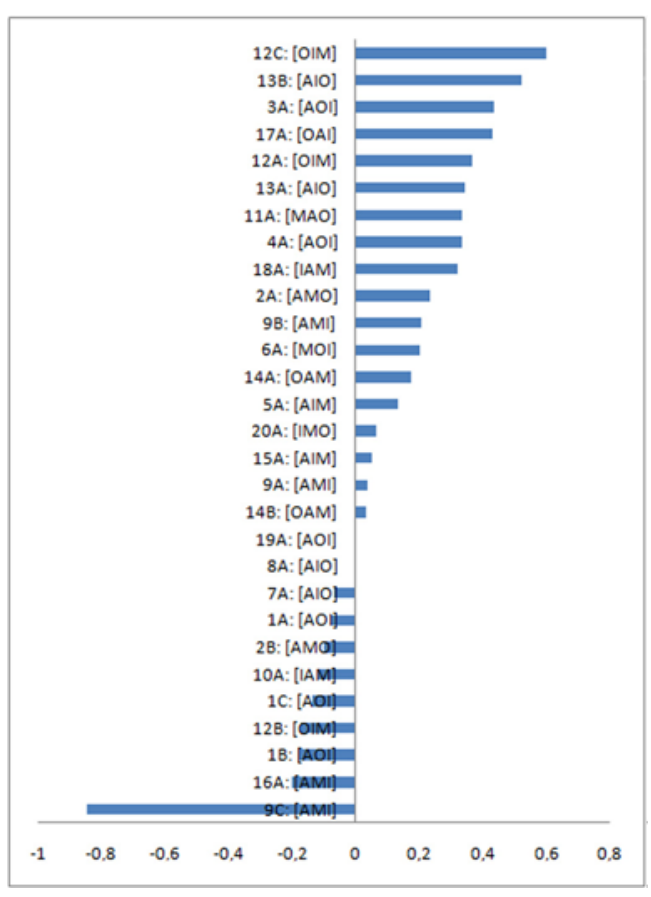

Fig. 4: Performance questions focused on how the requirements meet the needs. Performance is represented by the CP coefficient, together with a classification of the three most dominant requirement categories
The performances of requirements are shown by the value of the CP coefficient, which subtracts the number of responses on the scale (1-5) from the number of responses on the evaluation scale (3-7) and divides this number by the overall number of assessments. If the resulting coefficient is negative, this requirement can be perceived as insufficiently met. Each requirement is also displayed with the classification of the three most numerous groups. The classes are listed in descending order by the number of frequencies gained. The combinations indicate the approximate structures of class distributions and also the nature of the requirements. For example, request No. $16 \mathrm{~A}$, from the appendix marked here as A16 - How satisfied are you with the links between the teaching and practical applications? is perceived as attractive (A), but some of the respondents considered it as a must be $(\mathrm{M})$, and the coefficient of performance $C P$ is -0.2 , which indicates lack of compliance. Question $C$ of requirement No. $9-$ How satisfied are you with the spaces for parking? is percieved as a very poor performance, is classified as attractive (A) and consequently as a must be (M). The number of evaluations was only $43 \%$ due to the frequent choice does not affect me, which means that many of the students are not concerned with parking needs. If the indifferent class is dominant, the next class in order becomes decisive. Requirement No. $20 \mathrm{~A}$ - How satisfied are you with the possibility of continuing for a master's degree after graduating as a bachelor of STM? is a case of the dominant indifferent class and must-be class as second in the order, and therefore if the requirement is not met it will evoke dissatisfaction among the students. If it is met, it will be regarded as self-evident and will not evoke any improvement in student satisfaction. Requirement No. 2 - Helpful approach of teachers, mainly question $\mathrm{B}-$ How satisfied are you with the study materials? with a significant contribution of must be evaluation, also need to be addressed.

\section{Choice-Based Conjoint Analysis}

Conjoint analysis is an universal tool which might be used in market research to determine a marketing strategy. It belongs to the group of multivariate statistical methods designed for the most exact market survey. Conjoint anlaysis was originally derived from the fields of mathematical psychology and psychometry. In contrast to compositional approaches, where each specific incentive attribute is assessed separately, the conjoint approach is based on a global assessment of the incentives representing products. Respondents evaluate a set of concepts in a questionnaire, and assign their preference to them. Each 
product is a concept characterized by a specific combination of parameters, or by the characteristic levels of important attributes, and respondents must make trade-offs between the properties when considering concept preferences, because they are evaluating complex objects, each of which represents a specific product as a whole. This way of questioning is more similar to real buying behavior. In compositional approaches, product properties are evaluated without the context of the simultaneous presence of other attributes. Respondents do not need to make any compromises during the evaluation, for example due to a limited financial budget, or they do not take into the account a combination of manufacturer brand and other properties. They do not evaluate the utility of a product, but only the separate features, and the resulting preference characteristic is often significantly distorted. Choice-based models emerge from Mc Fadden's economic theory [7]. According to this theory, individuals and homogeneous groups of individuals produce the market behavior generated by maximizing preferences, which may include a random component due to fluctuation in perceptions, and attitudes and also the action of other immeasurable factors. Demographic, economic and social variables also shape the resulting preferences. Choice-based conjoint analysis (CBC) [8] is a specific approach from the group of conjoint methods. It is derived from Random Utility Theory (RUT), which is an aspect of more general Behavioral Theory. This approach focuses directly on the process of customers' purchasing decisions. A discrete choice qustionnaire also maintains hypothetical products, as in the traditional conjoint approach, but here respondents always choose only one concept per question. The question is formed as a subset of the total set of hypothetical concepts. In marketing and economics, the most important random utility model is the discrete-choice model (the choice of one option at the time). The data can be analysed using the COUNT method, which simply expresses the number of cases when selected concepts included the levels as a percentage of the total number of occurrences of these levels in a questionnaire. Other approaches for analyzing choice-based data are Multinomial Logit (MNL), Multinominal Probit (MNP), Latent Classes and MNL Hierarchical Bayes (HB).

The output of this method usually computes the probability that a particular type of respondent will buy a specified product (a combination of product features), or it determines the preferences of the market segment.

\section{Conclusion}

This article has presented issues of buying decision modeling in the context of the original motivation theories from psychology. An electronic survey was conducted among respondents belonging to a group of students at a technical university. A Kano Model was constructed for the identified requirements of students which were established during the intial stage. The model was analyzed. This allowed us to classify the requirements into the typical categories (attractive, one-dimensional, and must be) affecting the satisfaction and dissatisfaction of customers in different ways. It helped us to understand how the requirements work to meet students' needs. The current performances and importance ${ }^{1}$ of the requirements were also measured. It was possible to deduce from the results strong and weak aspects in the services related to education and to define potential improvements and new services that will have the greatest benefit for students.

A weak point in compositional methods lies in obtaining responses on individual attributes of a product or a service independently of other attributes. Products are offered in the market as bundles of attributes. Compositional methods are not able to capture the effect of combinations of attributes and are therefore often inaccurate. Conjoint analysis deals successfully with this issue. Its Choice-Based approach, tailored for modeling purchasing decisions, was introduced in Section 3.

\section{$\operatorname{Appendix}^{2}$}

1. Meals, housing and social facilities

A. How satisfied are you with the food?

B. How satisfied are you with the accomondation?

C. How satisfied are you with the sanitary facilities (toilets, etc)?

2. Helpful approach of teachers

A. How satisfied are you with the helpfulness of the teachers?

B. How satisfied are you with the study materials?

3. Good organization of lessons, optimal schedule and the need to move from place to place

A. How satisfied are you with the arrangement of your schedule?

4. Better opportunities through the image of the university

A. How satisfied are you with the offers of employment for graduates from the faculty?

\footnotetext{
${ }^{1}$ The measurement of requirement importance is not shown in this paper.

${ }^{2}$ The annex contains a list of requirements used in the survey to conclude the Kano model. The first level in the list retains the identified requirements; the second level contains the questions to investigate current performances.
} 
5. Top equipment at the faculty for teaching or research work

A. How satisfied are you with the support facilities used in teaching at the faculty?

6. Content accordance of the field of study with world trends

A. How satisfied are you with the appeal of your field of study?

7. Greater freedom in choice of curriculum

A. How satisfied are you with the current offer of courses?

8. Opportunity to gain a recognized certificate for language skills

A. How satisfied are you with the provisions for learning a foreign language?

9. Spaces available in the university for various purposes (sports, study halls for team discussions, etc)

A. How satisfied are you with the spaces for self study?

B. How satisfied are you with the sports facilities?

C. How satisfied are you with the spaces for parking?

10. Opportunities for scientific cooperation with the department during the studies

A. How satisfied are you with the opportunities to participate in projects carried out by the departments?

11. Availability of current information, and opportunities to use a uniform and comprehensive IS

A. How satisfied are you with the opportunities to obtain current information related to your studies?

12. Comfortable work with the KOS system and good interaction with the staff of the study department

A. How satisfied are you with the use of the KOS system?

B. How satisfied are you with office hours at the study department?

C. How satisfied are you with the behavior of the study department staff?

13. Use of the latest technology for teaching and opportunities to use your own notebook

A. How satisfied are you with the technical aids used in your classes?

B. How satisfied are you with opportunities to use your own laptop in your classes?

14. Compliance of the curriculum with your ideas about the field that you study

A. How satisfied are you with the range of required courses?

B. How satisfied are you with content of the individual courses?

15. Opportunities to study abroad
A. How satisfied are you with the offers for studying abroad?

16. The interrelation between teaching and practice

A. How satisfied are you with the links between teaching and practical applications?

17. The image and positive evaluation of the faculty in the world, its general recognition and reputation

A. How satisfied are you with the reputation of the University among the public?

18. Opportunities to choose interdisciplinary fields of study (a combination of fields)

A. How satisfied are you with the offer of courses from other fields of study?

19. Providing a wide range of student discounts

A. How satisfied are you with the offer of student discounts?

20. The existence of an opportunity to continue to a master's degree focused on the field of Software Technology and Management (STM)

A. How satisfied are you with the possibility of continuing to study for a master's degree after graduating as a bachelor of STM?

\section{References}

[1] Kotler, P., Keller, K.: Marketing Management. 13th ed. Pearson Prentice Hall, 2009.

[2] Reynolds, T. J., Gutman, J.: Laddering theory, method, analysis, and interpretation. Understanding Consumer Decision Making-The MeanEnd Approach to Marketing and Advertising Strategy, p. 25-62.

[3] Biligili, B., Ünal, S.: Kano Model Application for Classifying the Requirements of University Students. MIBES, 2008, p. 31-45.

[4] Sauerwein, E., Bailom, F., Matzler, K., Hinterhuber, H.: The Kano model: How to delight your customers. International Working Seminar on Production Economics, 1996, vol. 1, p. 313-327.

[5] Shiba, S., Graham, A., Walden, D., Lee, T. H., Stata, R.: A new American TQM: Four practical revolutions in management. Productivity Press Portland, Ore, 1993.

[6] Berger, C.: Kano's methods for understanding customer-defined quality. Center for Quality of Management Journal, 1993, vol. 2, no. 4, p. 3-36.

[7] McFadden, D.: The Choice Theory Approach to Market Research. Marketing Science, 1986, vol. 5, no. 4, p. 275-297.

[8] Orme, B.: The CBC System for Choice-Based Conjoint Analysis. Sawtooth Software, Inc., 2008, p. 1-27. 


\begin{abstract}
About the author
Ondrej Grünwald was born in Karlovy Vary in 1978. He received his Ing. degree in 2008 from the Faculty of Electrical Engineering of the Czech Technical University in Prague, specializing in Economics and Management in Electrical Engineering. Currently he is a Ph.D. student at the Department of Economics, Management and Humanities at the Faculty of Electrical Egineering of CTU in Prague. His interests include the application of mathematical
\end{abstract}

and statistical methods in marketing, mainly conjoint analyses and related techniques for marketing research.

Ondřej Grünwald

E-mail: grunwond@fel.cvut.cz

Dept. of Economics

Management and Humanities

Czech Technical University

Technická 2, 16627 Praha, Czech Republic 